engagement and recovery within medical treatment plans. Stimulants (e.g. methylphenidate) are first line licensed medications for $\mathrm{ADHD}$, and selective norepinephine reuptake inhibitors (e.g. atomoxetine) and alpha-2A adrenergic receptor agonists (e.g. guanfacine) are second line. At least $85 \%$ of children respond to stimulants, and only require alternative medications due to poor tolerance profiles.

European Union member states classify narcotics according to United Nation conventions, and stimulant medications are classified as controlled drugs. This means that they have strict prescribing rules, which if not adhered to results in delayed dispensing, continued impairment, and increased demands on already stretched clinical resources. In Ireland ADHD is treated by tertiary mental health services, however in many European countries, paediatricians take a lead role in ADHD treatment.

Aim This study examines the knowledge of trainee doctors in Ireland of the prescribing restrictions of stimulant medications, and the alternative non controlled medications used to treat ADHD.

Method A questionnaire was designed to capture the current knowledge and prescribing habits of Irish trainee doctors on the 4 commonly used ADHD medications i.e. methylphenidate based, amphetamine based, atomoxetine and guanfacine. The percentage of correct answers were calculated, and Chi squared testing used.

Results 47 questionnaires were completed and returned with a response rate of $57.5 \%$. The majority of doctors knew that stimulants were controlled and how to prescribe these, however only one third of doctors distinguished correctly that the other 2 drug classes were not controlled, and similarly did not know how to prescribe them.

Conclusion There is a knowledge gap amongst trainee doctors around prescribing for ADHD. Mainly for the non-controlled medication options, but also on specific points around prescribing the controlled stimulant medications. We hope that this identified gap in knowledge can be filled with targeted teaching; supporting doctors to become informed, competent and comfortable in prescribing for this common childhood illness.

\section{P171 ANTENATAL CORTICOSTEROID THERAPY: TO DETERMINE THE LEVEL OF ADHERENCE TO PROTOCOL IN PRETERM NEONATES}

Sarah Kasha*, Aliya Hamid, Brian Fox, Michael B O'Neill. Mayo University Hospital, Castlebar, Ireland

\subsection{6/archdischild-2019-epa.526}

Background and aim Institute of Obstetricians and Gynaecologists (RCPI) guideline for preterm prelabour rupture of the membranes (PPROM) recommends the administration of antenatal corticosteroids for women who are 24 to 36 weeks pregnant with anticipated preterm labour. It reduces the risks of respiratory distress syndrome, intraventricular haemorrhage and necrotizing enterocolitis. Recommended steroid is $12 \mathrm{mg}$ of intramuscular Betamethasone given 24 hours apart and at least 24 hours before delivery. This study determined the level of adherence to the guidelines.

Methodology Mothers who presented in preterm prelabour rupture of membranes between $1 / 1 / 2015$ and 31/12/2018 were incorporated into this study. Mothers were evaluated for maternal age, parity, body mass index, ethnicity and demographic distribution. Babies were evaluated for gender, gestational age, weight, corticosteroid type and time of administration prior to delivery, number of doses, type of respiratory support, comorbidities and length of hospital stay. Data was recorded on a collection sheet.

Results 103 mothers with PPROM were eligible to receive antenatal steroids, of whom 44(43\%) received recommended treatment and 59(57\%) received incomplete or no treatment. Out of infants who received complete course of antenatal steroids, $7(16 \%)$ required surfactant and intubation, 3 (7\%) required CPAP and surfactant, $16(36.4 \%)$ required only CPAP, 1 (2\%) required high flow oxygen and 17(38.6\%) did not need any respiratory support. Of the 59 infants who had an incomplete course or no steroids, $12(20 \%)$ required surfactant and intubation, $4(6.8 \%)$ required surfactant and CPAP, 20 (34\%) required CPAP, 1 (2\%) required low flow oxygen and $22(37.2 \%)$ did not require any support. Of those who received complete treatment, 26 were early preterm $(28-<34$ weeks) and 20 were late preterm $(34-34+5$ weeks). Of those who received incomplete or no treatment, 28 were early preterm and 29 were late preterm. The mean gestational age for infants who received complete treatment was $30 \pm 2$ weeks, and for those who received incomplete treatment was $32 \pm 2$ weeks. Of the 103 mothers, 88(85.4\%) were Irish and 15 (14.6\%) were Non-Irish.

Conclusion It was found that adherence to guideline is satisfactory. Imminent preterm delivery prevented $100 \%$ adherence to the protocol. This is a risk reduction strategy and parents should be made aware of the possible outcomes.

\section{P172 TRENDS IN PAEDIATRIC MORBIDITY AND MORTALITY AT UNIVERSITY HOSPITAL LIMERICK}

Eoin Ryan*, Sarah Ann Bennett, Emma Dunne, Eoin Fitzgerald, Erica Crothers, Cillian Lineen, Niofa Canty, Abrar Haider, Katie Flinn, Husnain Mahomed, Alwyn Charles, Siobhan Gallagher, Anne-Marie Murphy. University Hospital Limerick, Limerick, Ireland

\subsection{6/archdischild-2019-epa.527}

Background Morbidity and Mortality $(\mathrm{M}+\mathrm{M})$ meetings are an important strand of good clinical governance in institutions committed to maintaining and improving the quality of patient care. In 2015, the Department of Paediatrics, UHL made a decision to establish a Paediatric $\mathrm{M}+\mathrm{M}$. The meetings were designed to allow structured presentation of complicated Paediatric cases in a multidisciplinary forum to facilitate open discussion in order to identify areas of improvement. Since then, meetings have been held at quarterly intervals between 1300 and $1400 \mathrm{hrs}$ on the last Friday of 'term'. Morbidity is defined as cases requiring transfer to a tertiary hospital and mortality as all cases of children who died under Paediatric care. To date $10 \mathrm{M}+\mathrm{M}$ meetings have been held.

Aims Our aim was to review the recent trends in Paediatric Morbidity and Mortality in our region

Methods Medical charts, Emergency Department notes and transfer letters are reviewed to ascertain the presentation, initial assessments, management and mode of transfer etc of patients deemed to meet the criteria for our $M+M$. The Tertiary centres are contacted to clarify outcomes. This data is then structured into a PowerPoint presentation by two Paediatric Trainees assigned to the task and presented to the Department at the $\mathrm{M}+\mathrm{M}$ meeting facilitating discussion on 'human factors' and ' systems failures ' when identified. 